due, not to a change in level of expression of the oncogene, but to a change in its coding sequence; this suggests that the cancer may have resulted, in part, from exposure to the kind of mutagen that can produce this particular change, a GC to TA transversion.

We may be seeing here the shape of things to come. Even if mutation of the oncogene was not actually one of the ratelimiting steps in that particular patient's progression to bladder cancer, it does seem that the requisite technology is fast approaching that will make it practicable to search through selected regions of the genome for every kind of textual aberration. One spectacular example of this is about to be reported elsewhere ( $P$. Leder, personal communication). Most B-cell lymphomas bear a translocation of the end of chromosome 8 into the region of one of the chromosomes concerned with antibody synthesis (chromosomes 2, 14 or 22). It has now been possible to isolate the region of DNA involved in this rearrangement and show that it contains the proto-oncogene called $m y c$, that was originally found in association with a chicken leukaemia and, in humans, is located on chromosome 8 . So here too is another human cancer arising apparently as the result of activation of an oncogene, in this case because the gene has become caught up in the active chromosomal region involved in antibody synthesis.

We seem, therefore, to be at one of those wonderful moments in science when many lines of research come together and much that was impossibly obscure suddenly seems plain. There are, however, one or two caveats that are well known to everyone engaged in this work but may not be immediately obvious to outsiders.

Natural carcinogenesis is a multistep process. Evidence for this comes from epidemiology (for example, the relation between incidence of lung cancer and the frequency and duration of cigarette smoking) and from laboratory experiments, and shows that the formation of a cancer requires several independent, ratelimiting steps. Thus, the provision of an active oncogene, whether by activation of a cellular gene through rearrangement or mutation or by viral infection, presumably corresponds to only one of a sequence of steps. Given that cancer arises from a sequence of steps, we would expect the first steps to be the ones that allow clonal expansion and free competition between cells, because this should then make all the other steps occur more efficiently and allow continuous selection of faster growing cells. But special provisions appear to have been made to prevent competition between individual cells in intact tissues. So a significant component of carcinogenesis in whole animals must be the production of whatever changes are needed in order to allow competition. If, for example, the first step in carcinogenesis has to be the liberation of cells from control by cell-cell interaction, we could not find this out by studying the transformation of cell lines which have, of course, already taken this step.

Transfection experiments with NIH 3T3 cells are open to other objections. Mice will develop cancer at the site of implanted plastic sheets, and it may be for this reason that their cells can readily be induced to grow profusely in tissue culture dishes. Guinea pigs (and probably humans) are not susceptible to such foreign body carcinogenesis, and their cells do not readily form immortalized cell lines. We could argue that the $3 \mathrm{~T} 3$ cell is already partway to becoming a cancer cell, and for that reason may not provide the right assay for certain kinds of tumorigenic DNA.

In transfection experiments only about one in a million cells acquire and express the exogenous DNA. This may mean that only cells already possessing a special genetic or metabolic alteration are competent to acquire foreign DNA. Such cells could be just those that have taken one of the rate-limiting steps towards cancer; if transfectibility is evidence of a step towards transformability then this step cannot be elucidated in these experiments. (Although transformation by viruses is very efficient, the viral reverse transcriptase could conceivably be making gene rearrangements much more frequent and, in that way, be by-passing what is normally a ratelimiting step.)

Most tumours studied so far, whether occurring naturally in humans or produced by $\mathrm{X}$ rays and chemicals in animals, do not yield DNA that is oncogenic in cell transfection experiments. So the assay, as presently constituted, is not detecting the genetic events responsible for many types of cancer. It is important to remember that transfection can only pick up changes that, in the genetic sense, are dominant; also it will only detect oncogenes which provide products that these particular recipient cells require for transformation (for cxample, the myc gene is not efficient at transforming NIH 3T3 cells); lastly, there is the requirement in these experiments (and in tumorigenesis by viruses) that the transforming genes are not too large to be transferred intact.

Above all, we need to keep in mind that natural human carcinogenesis is a multistep process. We do not know what the rate-limiting steps are, nor which of them are most sensitive to environmental influences and therefore a proper object for preventative strategies. But we can at least feel that the technology for studying genes and their patterns of expression are now advancing so fast that the molecular biologist will soon be telling the epidemiologist what to look for.

\title{
100 years ago
}

THE PROJECTION PRAXINOSCOPE M. GASTON Tissandier describes in La Nature an ingenious adaptation of the praxinoscope, under the above name, by means of which the images are projected on a screen, and are visible to a large assembly. Our engraving will give an idea of the arrangement and the effect produced. By a modification of the "lampascope", M. Reynaud, the inventor, obtains by means of an ordinary lamp, at once the projection of the scene or background by the object-glass which is seen at the side of the lantern - and of the subject, by another object-glass which is shown in front of and a little above the same lantern. For this, the positions of phases which form a subject are drawn and coloured on glass, and are connected in a continuous band by means of any suitable material. One of these flexible bands is placed in the wide crown of the praxinoscope, which is pierced with openings corresponding to the phases of the subject. A hand-lever on the foot of the instrument allows a moderate and regular rotation to be communicated.

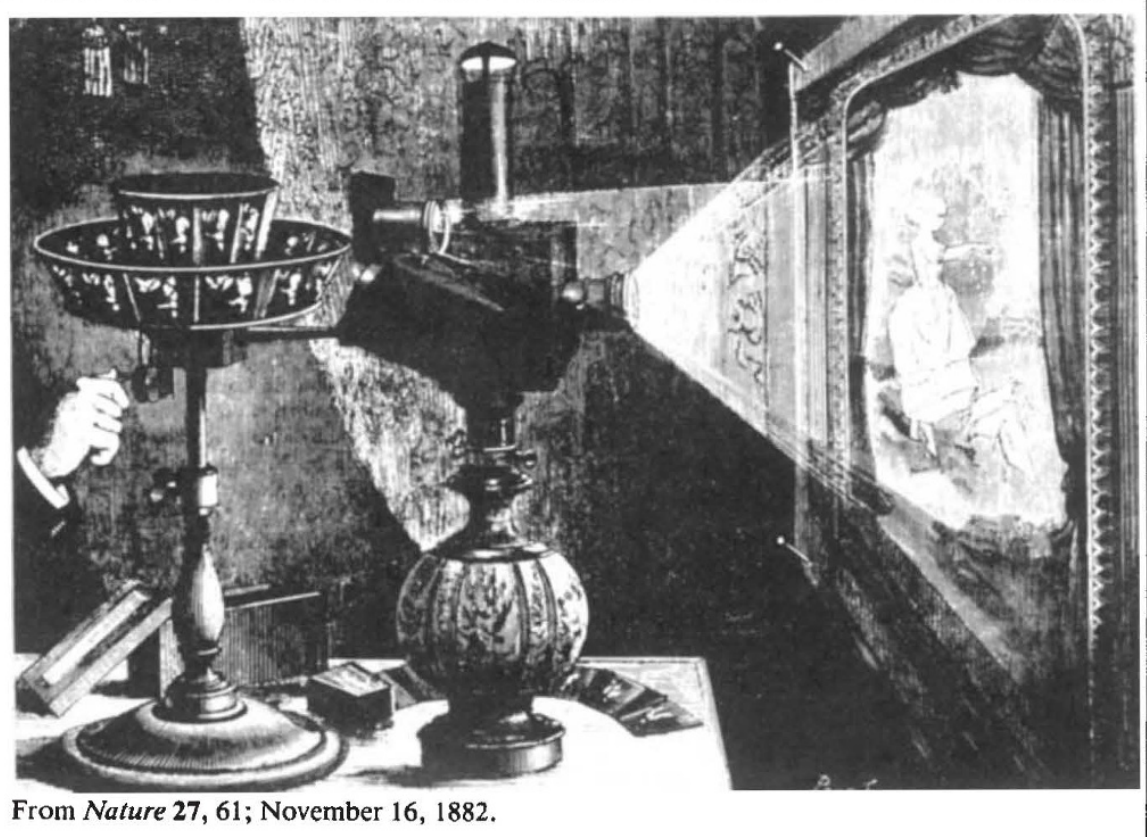

